Juli 2021 | innsbruck university press, Innsbruck

ZDS | ISSN 2791-4313 | www.zds-online.org

Nr.1/2021| DOI 10.15203/ZDS_2021_1.09

Lizenz: CC BY-ND 4.0 (Namensnennung, keine Bearbeitung)

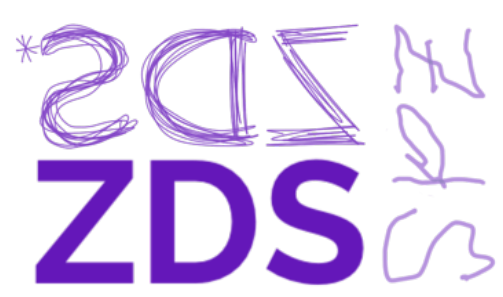

Bildnachweis: Steven Solbrig

Steven Solbrig

\title{
„Finger weg“ - Eine Sequenz aus Selbstporträts
}

\section{Zusammenfassung}

Was ist eine Hand? Was ist Behinderung? Mit welcher Perspektive Blicken wir auf beides im Alltag? Gehen solch "schwierige“ (O-Ton Fotografieprofessor) Fotos heute eigentlich noch in Zeiten von mehr und mehr vereffizienzierten Körperpolitiken? Wie sind die Reaktionen, bspw. von Menschen ohne Behinderung? Warum gibt es Künstler*innen mit Behinderung, die ihre Arbeit gern als „Disability Arts“ labeln würden, es aber nicht schaffen? Und hilft das beuys'sche Credo „Zeige deine Wunde!“ eigentlich noch in austeritäts-/ identitäspolitschen Zeiten?

Schlüsselwörter: Disability Arts

\section{"Hands off" - A Sequence of Self-Portraits}

\section{Abstract in English}

What is a hand? What is disability? With which perspective do we look at both in everyday life? Do such "difficult" (quote of a photography professor) photos still work today in times of efficient body politics? What are the reactions, for example, of people without disabilities? Why are there artists with disabilities who would like to label their work as "Disability Arts", but can't? And does Beuys' credo "Show your wound!" still help in times of austerity/identity politics?

Keywords: Disability arts 
ZDS: Deine Foto-Arbeit heißt: „Finger weg!“ Was bedeutet das? Befinden wir uns mit Deinen Fotos auf geheimem, verbotenem Terrain?

Steven Solbrig: Meine rechte Hand wurde 1986 in der ehemaligen DDR als „Anomalie“ eingestuft und ich landete daraufhin in der Kategorie "schwerbeschädigt". Noch heute zähle ich gesetzlich als „schwerbehindert". Dennoch „fehlen“ mir aus der Sicht vieler Nichtbehinderter „eigentlich nur" vier Finger. Mit dieser Serie möchte ich mich den Blicken von außen auf (meine) Behinderung widmen. Es ist für mich erstaunlich, wie sehr auch mein eigener Blick von der Mehrheitsgesellschaft geprägt ist. So finden sich in der Serie zum Beispiel Verweise auf Da Vincis Hände in „Die Erschaffung Adams". Außerdem wird auf diese schrecklichen Witze à la „Wie viele ... es braucht, um eine Glühbirne zu wechseln?“ angespielt. Zu sehen ist auch die ruhende Hand des werdenden Vaters auf dem Bauch der schwangeren Frau, die natürlich ein „gesundes“ Kind erwartet. Für mich DAS Klischeefoto der (Hetero-)Normativität.

ZDS: In Deiner Motivauswahl spielst Du mit Licht und Schatten, mit Spiegelungen und Doppelungen. Was bedeutet das, gibt es da für Dich einen Zusammenhang zum Thema Behinderung?

Steven Solbrig: Der Begriff der Fotografie bedeutet im wortwörtlichen Sinn „Schreiben mit Licht“. Deshalb ist mir wichtig, dass sich in meiner Arbeit schon ein gewisser künstlerischer Anspruch verorten lässt. So versuche ich, mit den verschiedensten fotografischen Stilmitteln den „Blick auf Behinderung" ein wenig zu erweitern. Denn ich finde, um die tatsächliche Vielfalt von Behinderung innerhalb der Gesellschaft sichtbar zu machen, muss da mehr her als "GdB [Grad der Behinderung]", „Disability Mainstreaming“ oder ein expliziter Rekurs auf das soziale Modell von Behinderung. Ich bin mir nicht sicher, ob es dafür unbedingt die „Disability Aesthetics", wie sie einst Tobin Siebers lancierte, oder gar ein Label der „Disability Culture“ braucht. Aber wir kämen beispielsweise in kulturpolitischen Debatten sicherlich ein Stück weiter, wenn wir, angelehnt an Anne Waldschmidt, uns fragen würden, auf welche Weise Behinderung als eine Form von Alterität durch die mehrheitsgesellschaftliche Kultur gegenwärtig reproduziert wird. Hierzu braucht es meiner Meinung nach Antworten, und das nicht nur von einzelnen Personen

ZDS: Was bedeutet für Dich die Hand in der Kunst? Was bedeutet es, wenn sie anders aussieht als üblich, wenn sie quer zu dem liegt, was von Händen erwartet wird?

Steven Solbrig: Puh, da gäbe es jetzt ganz viel zu sagen, vor allem aus kulturwissenschaftlicher Perspektive. Ich möchte jedoch viel lieber versuchen, mit ein paar Zeilen aus Lezlie Fryes „Things You Don't Think About When You've Got Ten Fingers“, über die ich einst bei der Lektüre von Rosemarie Garland Thomson stolperte, zu antworten: „Into submission into/Wishin“I was someone who looked a different way in this/ Ten finger world/ Ten fingers swirl around me as I learn to say, Wait for me/You can wait while I see what I can do with my body, with my hands."

\section{Künstler_in}

Steven Solbrig, weiß, gender questioning, mit Behinderungseinrichtungs- sowie Ost-Erfahrung, arbeitet seit einigen Jahren kulturpolitisch zur Sichtbarkeit und Teilhabe von Künstler_innen mit Behinderung. Als Teil des Performanceduos „Jane Blond and that Stevil Kniewel“ arbeitet Steven Solbrig derzeit performativ zu Assistenz, Care und Pflege aus der Sicht von Personen mit Behinderung.

E-Mail: post@stevensolbrig.de; Homepage: https://stevensolbrig.wordpress.com/ 


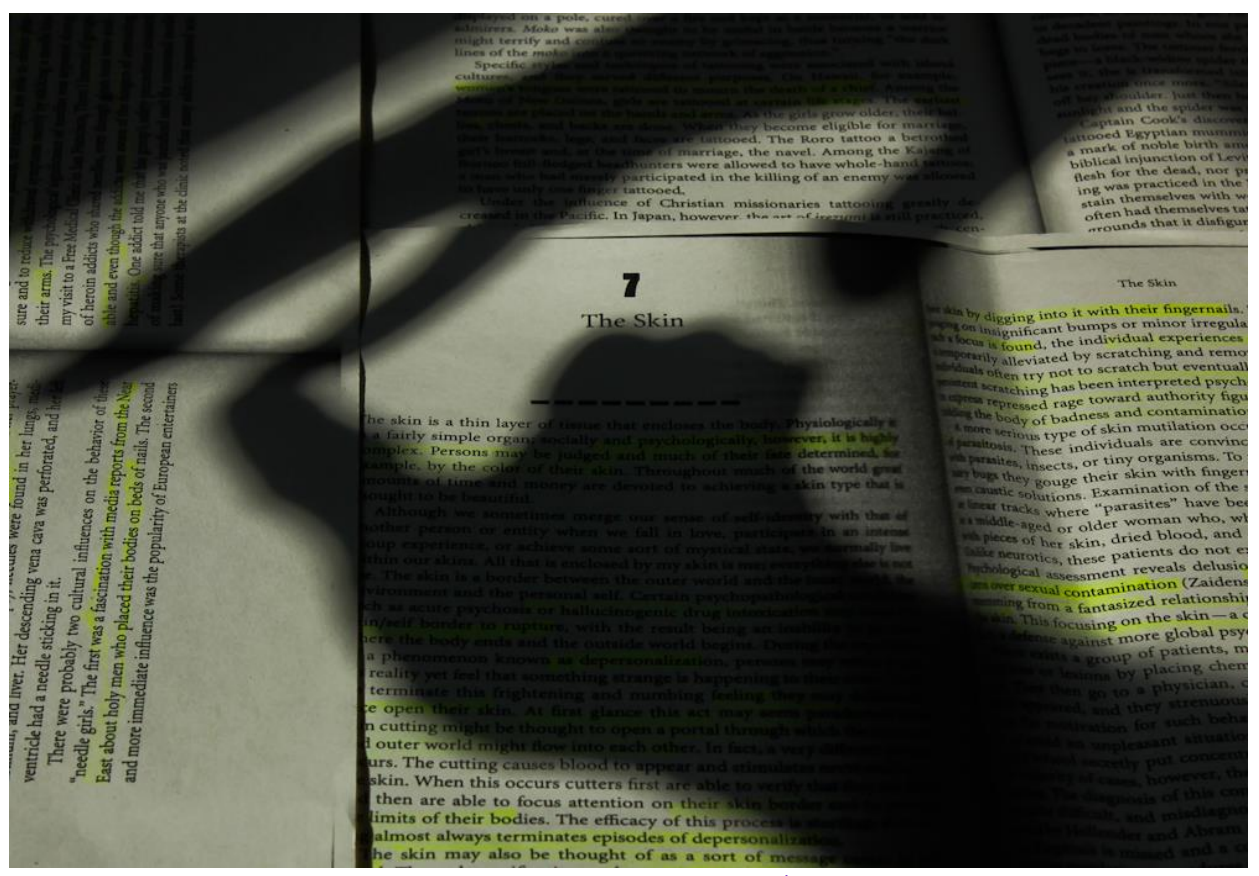

Abbildung 1: \#1 (Quelle: Steven Solbrig/ CC BY-ND 4.0)

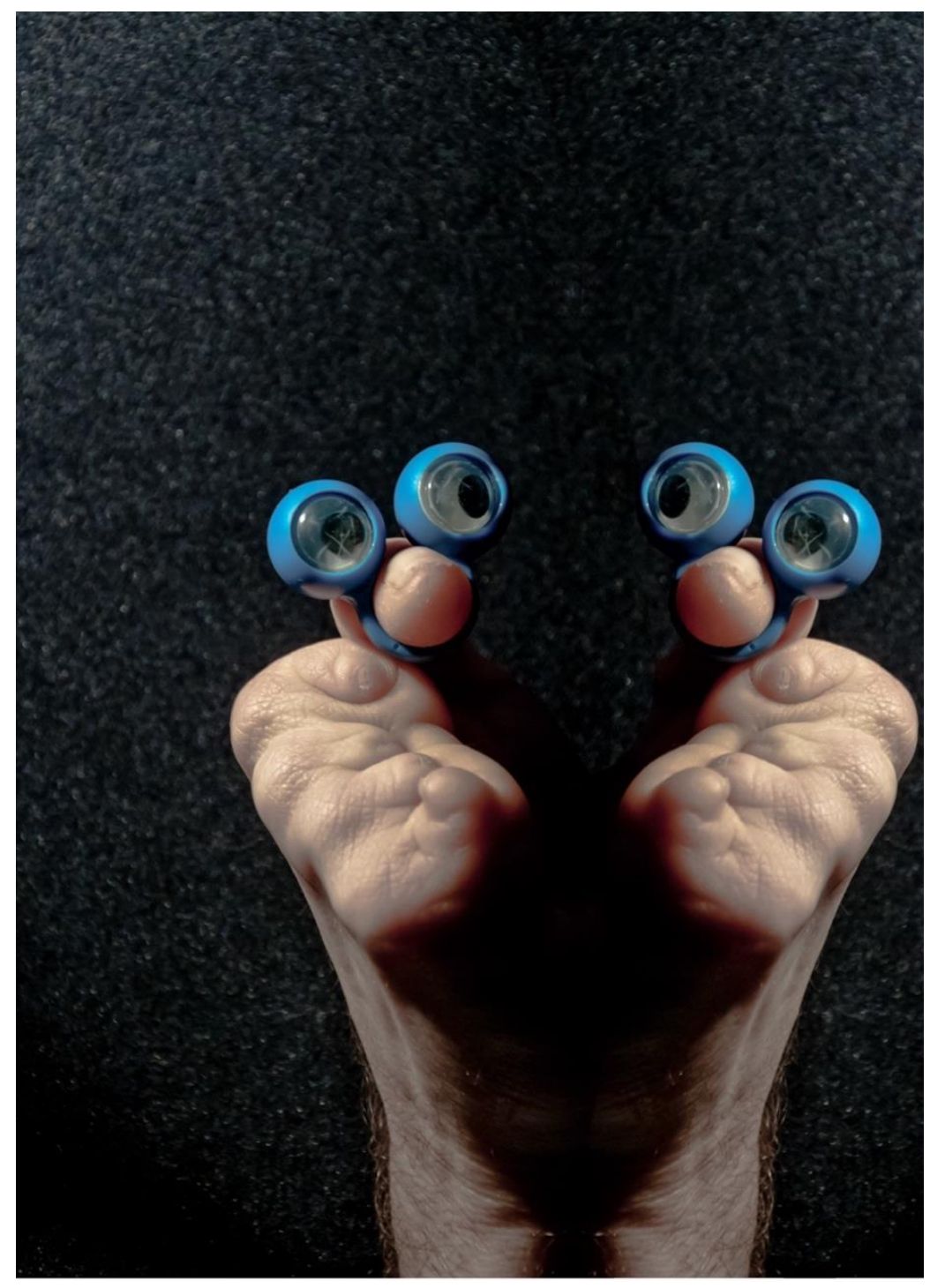

Abbildung 2: \#4 (Quelle: Steven Solbrig/ CC BY-ND 4.0) 


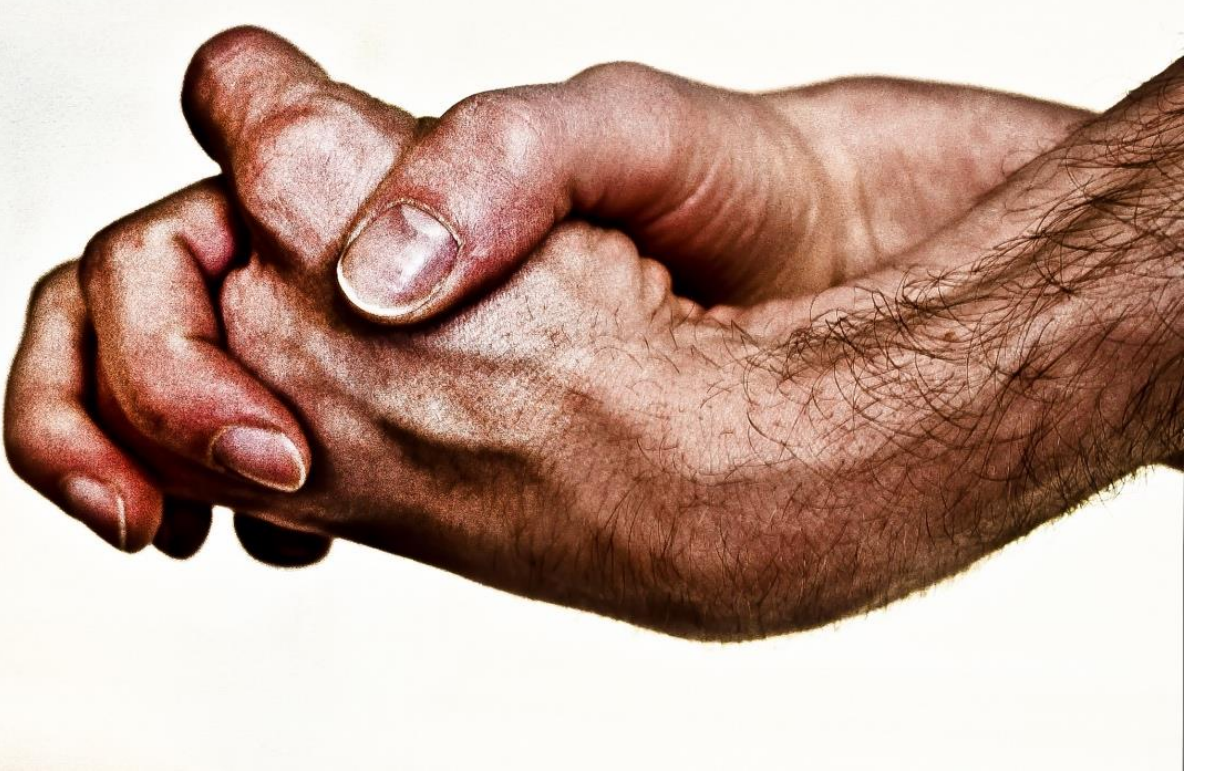

Abbildung 3: \#5 (Quelle: Steven Solbrig/ CC BY-ND 4.0)

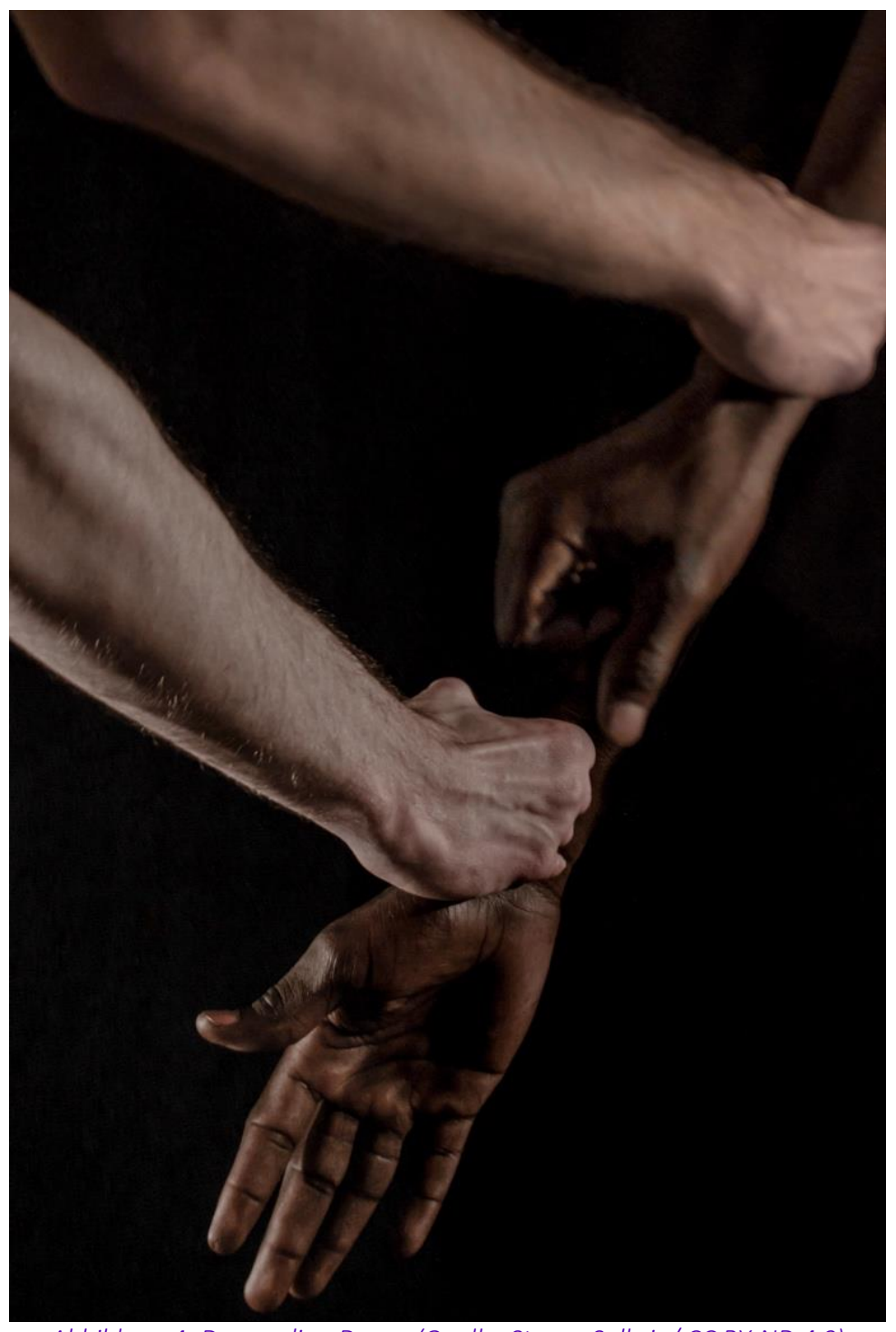

Abbildung 4: Demanding Dance (Quelle: Steven Solbrig/ CC BY-ND 4.0) 


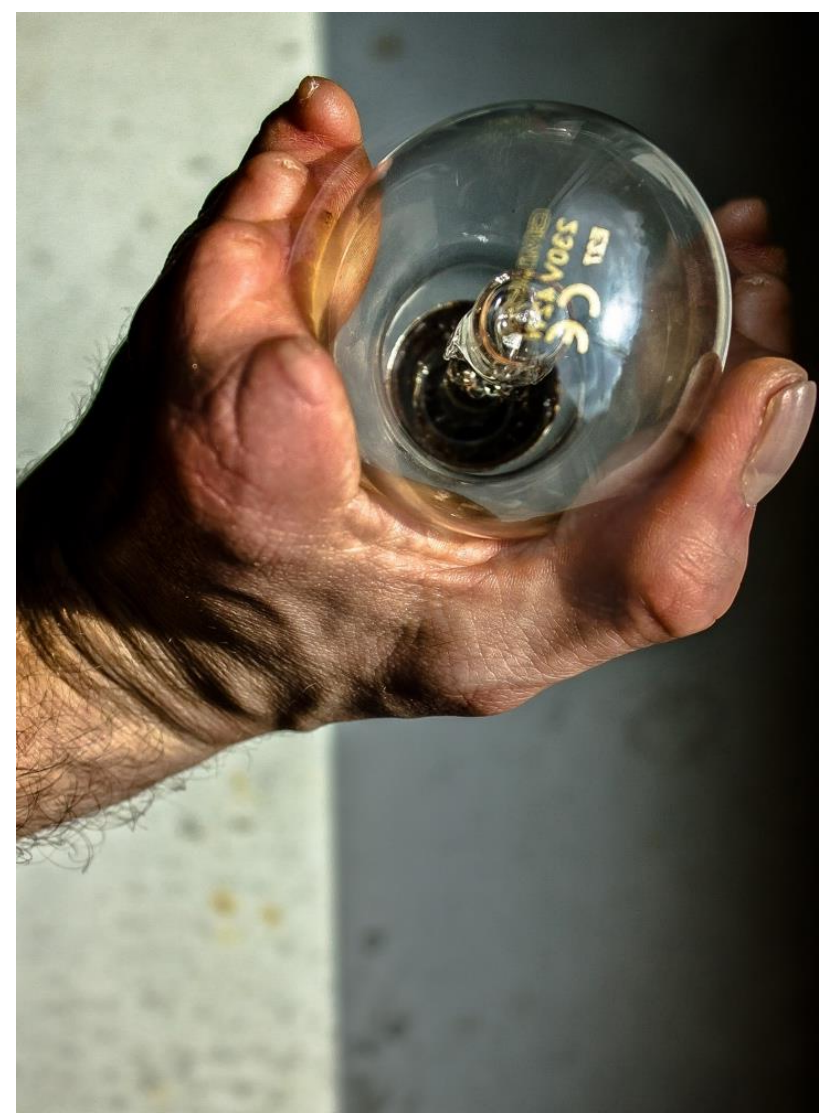

Abbildung 5: \#8 (Quelle: Steven Solbrig/ CC BY-ND 4.0)

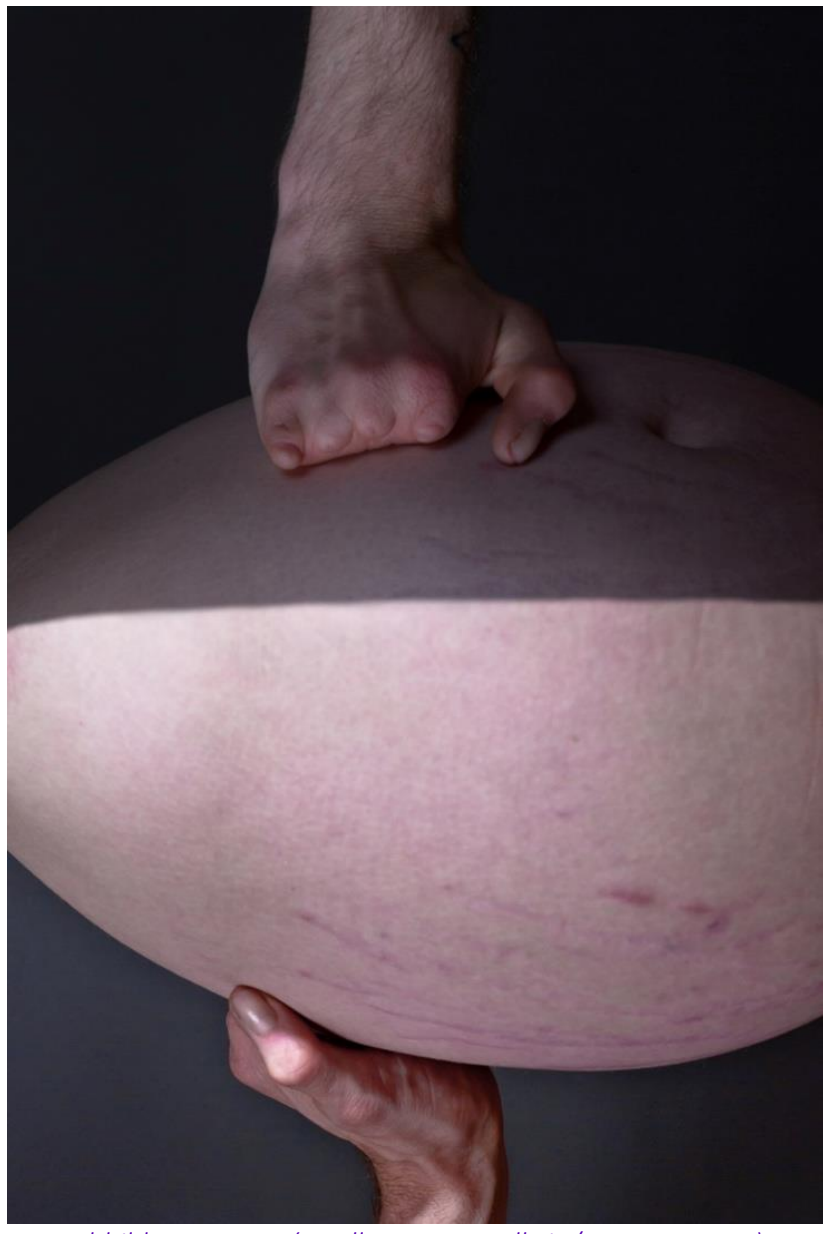

Abbildung 6: \#11 (Quelle: Steven Solbrig/ CC BY-ND 4.0) 